\title{
INDOOR SUBSPACING TO IMPLEMENT INDOORGML FOR INDOOR NAVIGATION
}

\author{
H. Jung ${ }^{\text {a }}$ J. Lee ${ }^{\mathrm{a} *}$ \\ ${ }^{a}$ Dept. of Geoinformatics, The University of Seoul, 163 Seoulsiripdaero, Seoul, Korea - (hyojin5919, jlee)@uos.ac.kr
}

Commission VI, WG VI/4

KEY WORDS: Subspacing, Indoor Space, Indoor Navigation, IndoorGML

\begin{abstract}
:
According to an increasing demand for indoor navigation, there are great attempts to develop applicable indoor network. Representation for a room as a node is not sufficient to apply complex and large buildings. As OGC established IndoorGML, subspacing to partition the space for constructing logical network is introduced. Concerning subspacing for indoor network, transition space like halls or corridors also have to be considered. This study presents the subspacing process for creating an indoor network in shopping mall. Furthermore, categorization of transition space is performed and subspacing of this space is considered. Hall and squares in mall is especially defined for subspacing. Finally, implementation of subspacing process for indoor network is presented.
\end{abstract}

\section{INTRODUCTION}

Finding a way through an indoor environment becomes an important factor in daily life as buildings have recently become more complex and spacious. For indoor navigation service, indoor information and relations of indoor space are needed. Considering efficient navigation service in indoor space, indoor network to implement IndoorGML has been constructed. In IndoorGML, all of indoor space which can be divided by physical constraint like construction elements (door, wall, window etc.) are set as a node and relation of these spaces are set as an edge (Lee, 2004). While this approach for applying IndoorGML into indoor navigation makes constructing indoor network easy, it is still not sufficient for providing navigation service in complex and large spaces. Presenting spacious areas like airport, shopping mall, or museum as a node can only provide simple network which show relations with large hall, lobby or corridor, yet indoor network fully understanding indoor environment having many obstacles like advertisement, or column is needed for indoor activities.

Recently, the attention in subspacing for indoor navigation increased. Zlatanova, Liu, and Sithole (2013) discussed a physical and conceptual framework for subspacing that can be used in any circumstances. They explain that this framework can be used for flexible context aware path-planning in indoor environment. They further explained that space subdivided not only geometrically but also semantic for providing better paths. In complex indoor environment, semantics is an important factor for multi-user and multi-purpose navigation. Conceptual model for determination of functional areas within indoor space and rules to divide indoor space into navigable and nonnavigable space is proposed by Krūminaite and Zlatanova (2014). Their conceptual model is focused on human perception of indoor environment and human social behaviour.

The above discussed article has focused on subspacing considering semantics. They considered circumstances of the indoor environment and human behaviour. The indoor network they proposed is not related to a real path, which is in the scope of this work. In order to provide a similar indoor network with real path, Khan and Kolbe (2012) discussed subspacing based on locomotion types (walking, driving and flying). This approach not only considers semantics of space but also constraints of specific locomotion type. Kang, Jung and Lee (2015) presented the subspacing strategy considering physical, logical and functional constraints to impelement IndoorGML. Figure 1 illustrates this process of subspacing in indoor space for creating applicable indoor network.

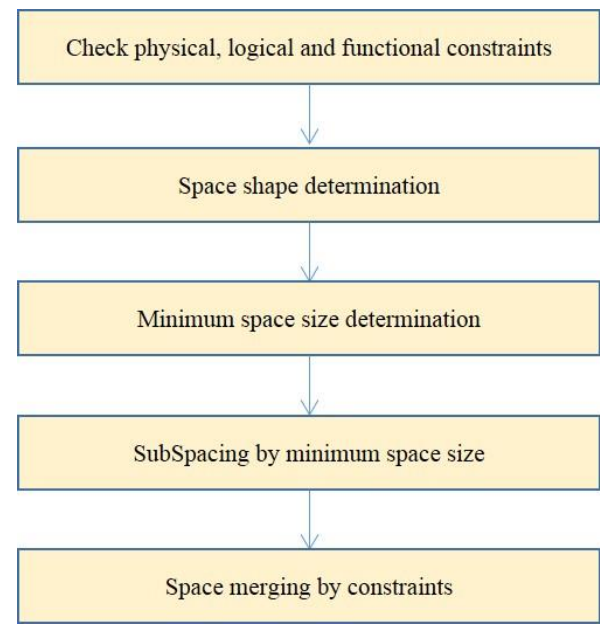

Figure 1. Process of Subspacing

Keeping in view the same idea, this work concentrates on the importance of subspacing to implement IndoorGML that are related to constructing applicable indoor network. It also explains a procedure to constructs in space for transition. Especially, subspacing method may be different according to place types like shopping mall, hospital or station. Thus, this study concentrates on how subspacing based on space types can be defined for indoor navigation in shopping mall.

\footnotetext{
* Corresponding author
} 


\section{SUBSPACING FOR CONSTRUCTING INDOOR NETWORK}

\subsection{Subspacing to implement IndoorGML}

IndoorGML introduces subspacing for indoor navigation. Subspacing refers to partitioning the space for constructing similar network with a geometrically optimal path. This approach can also represent topological relation (connectivity, accessibility) of indoor space by NRG. Figure 2 illustrates how to subdivide the space to implement IndoorGML for indoor network.

Given the geometric model, all of room is divided by geometric property according to NRG (figure 2-(b)). Concerning the node in hall, it is converted into 2 nodes for logical presentation. Therefore, transition space like hall, or corridor have to be concerned for subspacing.

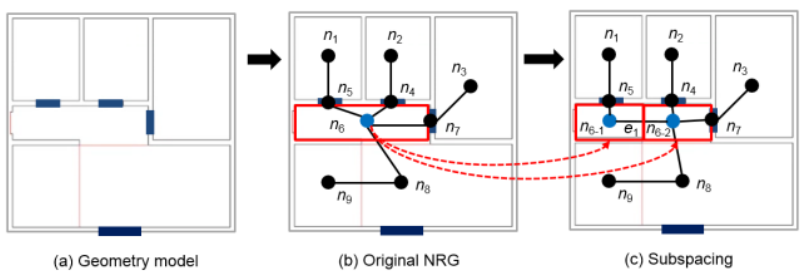

Figure2 Example of subspacing

\subsection{Categorization of Space types in Subspacing for Indoor Navigation}

Indoor space can be categorized by their function: Stay space or transition space. Stay space is room where people want to stay like the toilet, a store or an office. As destinations for indoor navigation are this kind of spaces, these spaces don't have to be partitioned. The term transition space refers to room which connects between other spaces like a corridor and a hall. To offer indoor navigation service, it is important to determine how to partition this kind of space to construct indoor network. However, subspacing in indoor environments differ with respect to the place types. Thus, we determine our target place as shopping mall. The transition space considered for this study is defined by the circumstances of shopping mall. The transition space types are defined as below.

Hall: Connects one space to another space like stay space, exit, or entrance. This concept contains a corridor and a passageway. In a shopping mall, stores are aligned along the hallway. The shape of this space is commonly rectangular. Direction of movement in this space is regular. People can move through the hall straightforward or enter the stores connected with hall.

Square: An open space where people can gather. This space intersects halls. In a shopping mall, a square is surrounded by stores. Shapes of this space are rectangle, square, or circle. The direction of movement is irregular. People in square can move at any direction.

\subsection{Constructing Indoor Network for indoor navigation}

Kang et al. (2015) have introduced a process of subspacing for applicable indoor network. Figure 23 illustrates how subspacing was performed in two steps for this purpose. First, defining target space is performed by physical, semantic, and functional constraints. Indoor environment is determined as navigable or non-navigable space by physical constraints. Then, accessibility and function of possibility is determined by semantic and functional constraints.

After defining the target space by physical, semantic, and functional constraints, subspacing is performed to transition space for constructing the network. This space is partitioned by subspaces for connecting to other transition space or stores. In case of a hall, the configuration of stores is considered. Each subspace connects to one door of space, yet doors which face each other are considered together. In case of a square, connectivity with store or halls is considered. As shape of square are irregular and people freely move in any direction, a square is partitioned as much smaller size of subspace than hall. When a hall connects with stores, subspaces which attach to stores have to contain one door of space. Then, stay space and subspaces are set as node. Edges connect the nodes presenting each space. Indoor network for indoor navigation is constructed by this process.

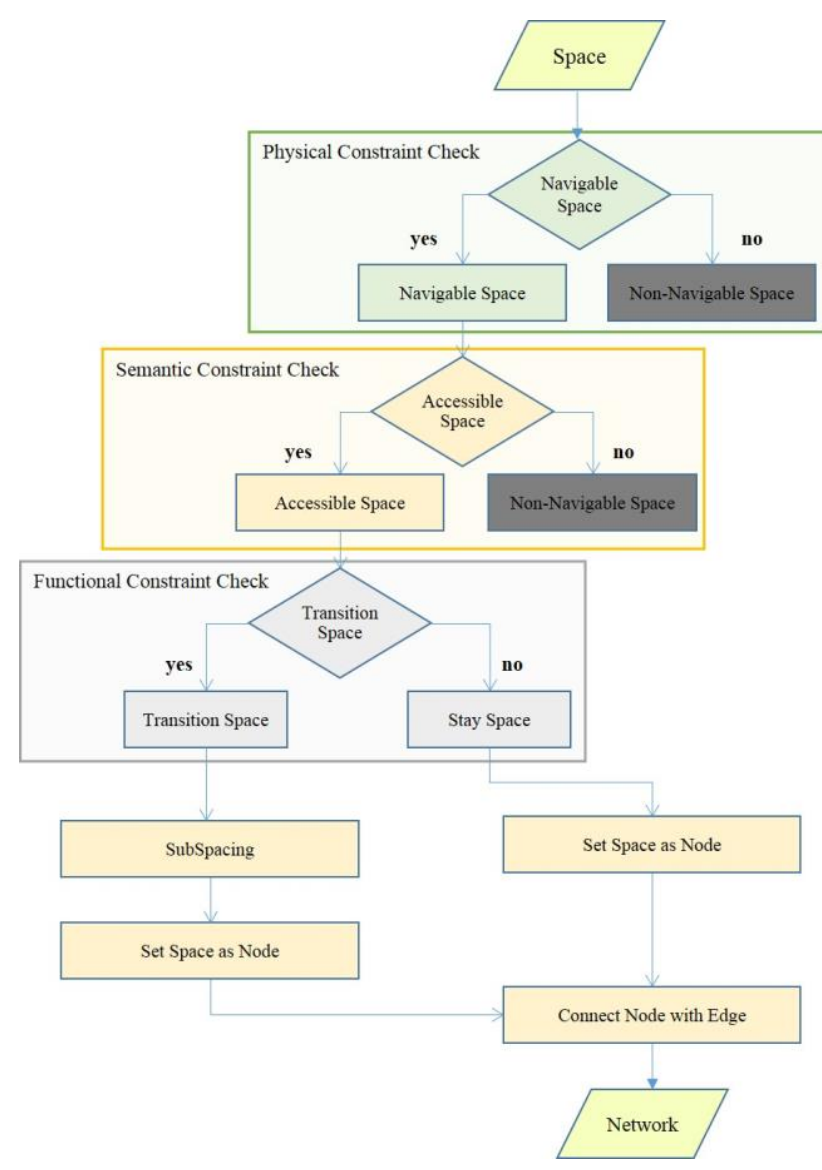

Figure 3. Flowchart for Constructing Indoor Network Process

\subsection{Implementation}

For subspacing indoor space based on transition space, we set Square 1 of COEX mall in Seoul as the target place. This space contains 47 stores, 2 restaurants, 1 square and 6 halls. The aim of this implementation is to offer appropriate indoor navigation service in shopping mall.

First, physical, logical, and functional constraints are applied into the target place. Stores and restaurants are categorized as a stay space, and a square and hall as transition space. Second, subspacing is performed. In this work, a grid is set as shape of subspace and $1 \mathrm{~m}$ is the size of the subspace, considering 
pedestrian's speed in indoor environment. Finally, subspaces are merged. Stay space is converted to a consolidated space. Transition space is merged considering the configuration of stores and connectivity. When merging space, visibility of pedestrians (Stoffel, Lorenz and Ohlbach, 2007) or function of space (Lee et al., 2011) can be applied. Stoffel et al. (2007)'s algorithm that partition the space into non-overlapping convex subspaces for visibility is used in this implementation. The navigation network was constructed by calculating centroid of subspaces and establishing links between centroids of neighbouring subspaces. Figure 4 denotes result of following subspacing process based on transition space.

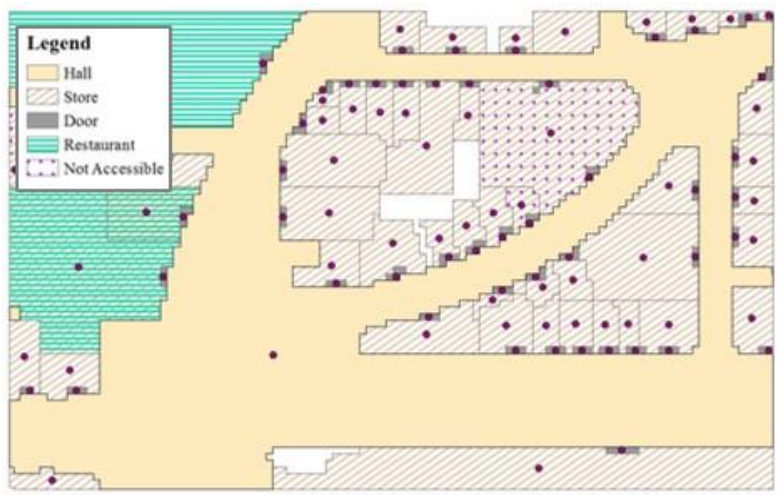

(a) Target Place
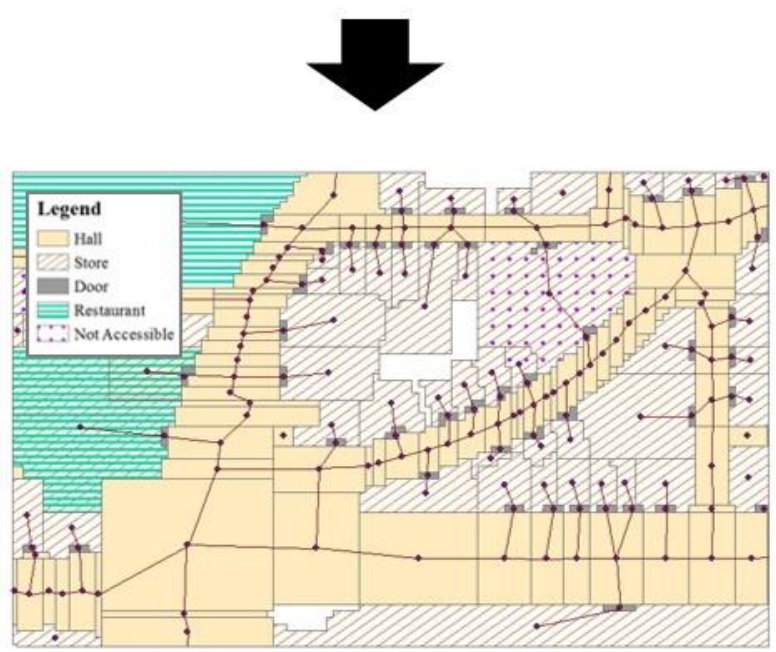

(b) Constructing Indoor Network

Figure 4. Result of subspacing based on transition space types in shopping mall (COEX mall)

\section{CONCLUSION AND FUTURE WORK}

We dicussed subspacing to implement IndoorGML in order to offer efficient indoor navigation service. Also, Categorization of transition space in shopping mall is performed. It can reduce the complexity of constructing indoor network. Furthermore, indoor navigation network is presented by a proposed subspacing process based on transition space types. In the future, we will focus on examining further constraints like opening hours, time, or user for subspacing considering indoor service. Moreover, additional research constructing indoor navigation network in other place types could be performed.

\section{ACKNOWLEDGEMENTS}

This research was supported a grant from geospatial information workforce development program funded by the Ministry of Land, Infrastructure and Transport of Korean Government (2014-04-09)

\section{REFERENCES}

Kang, H. Y., Jung, H., and Lee, J., 2015. A study of subspacing strategy for service applications in indoor space. Journal of Korea Spatial Information Society, 23(3), pp.??-??

Khan, A. A., and Kolbe, T. H., 2012, Constraints and their role in subspacing for the locomotion types in indoor navigation. In: Proc. the International conference on indoor positioning and indoor navigation, Sydney, Australia, pp. 1-12.

Krūminaitè, M. and Zlantanova, S., 2014. Indoor space subdivision for indoor navigation, In: Proc. The sixth ACM SIGSPATIAL International Workshop on Indoor Spatial Awareness, Dallas, USA, pp. 25-31.

Lee, J., 2004. A spatial access-oriented implementation of a 3-D GIS topological data model for urban entities. Geoinformatica, 8(3), pp.237-264.

Lee, S. J., and Lee, J., 2011. Navigable space-relation model for indoor space analysis. Journal of Geographic Information System Association of Korea, 19(5), pp. 75-86.

OGC, 2014, Indoor Geography Markup Language (IndoorGML) Enconding Standard, http://docs.opengeospatial.org/is/14-005r3/14-005r3.html (30 Jul. 2015)

Stoffel, E. P., Lorenz, B. and Ohlbach, H. J., 2007. Towards a semantic spatial model for pedestrian indoor navigation. In: Advances in Conceptual Modeling-Foundations and Applications, pp. 328-337

Zlantanova, S., Liu, L., and Sithole, G., 2013. A conceptual framework of space subdvision for indoor navigation. In: Proc. the fifth ACM SIGSPATIAL International Workshop on Indoor Spatial Awareness, Orlando, USA, pp. 37-41.

Revised July 2015 